DOI: 10.34185/1991-7848.itmm.2020.01.044

УДК 519.6

\title{
ХАОТИЧЕСКИЕ КОЛЕБАНИЯ В RLD ЦЕПЯХ
}

\author{
Деревянко А.И. к.т.н., доцент \\ Национальная металлургическая академия Украины
}

Ключевые слова: ХАОТИЧЕСКАЯ ДИНАМИКА, RLD ЦЕПИ, ТОЧКИ БИФУРКАЦИИ.

Целью работы является разработка математической модели RLD цепей и исследование влияния частоты $w$ и амплитуды $U$ входного гармонического напряжения на максимального значение напряжения $U_{d}$ на полупроводниковом диоде.

Хаос - это непериодическое поведение детерминированных нелинейных динамических систем, которое чувствительно к начальным условиям. Одним из сценариев перехода к хаосу является удвоение периода [1].

Простая схема, которая может демонстрировать хаотическое поведение, является так называемой схемой RLD, образованная последовательным соединением резистора $\mathrm{R}$, катушки индуктивности L и диода $\mathrm{D}$.

B случае доминирования барьерной емкости диода за счет выбора соответствующей рабочей точки, такая RLD цепь описывается уравнением

$$
L \frac{d^{2} q}{d t^{2}}+R \frac{d q}{d t}+\frac{A}{b} q^{\frac{1}{1-\gamma}}-\frac{1}{b}=U \sin w t
$$

Представленное уравнение (1) можно отнести к классу уравнений Дуффинга, отличительной особенностью которых является существование разнообразных периодических решений исследованных весьма мало, а еще в меньшей степени изучены непериодические решения [2].

Для решения поставленной задачи была использована среда моделирования Multisim, т.к. она обеспечивает интерфейс, приближенный к реальной аппаратной среде исследования реализаций электронных схем. Кроме того, в ней представлен широкий спектр элементов электронных схем с подробными и точными характеристиками. 
International scientific and technical conference Information Technologies in Metallurgy and Machine building - ITMM 2020

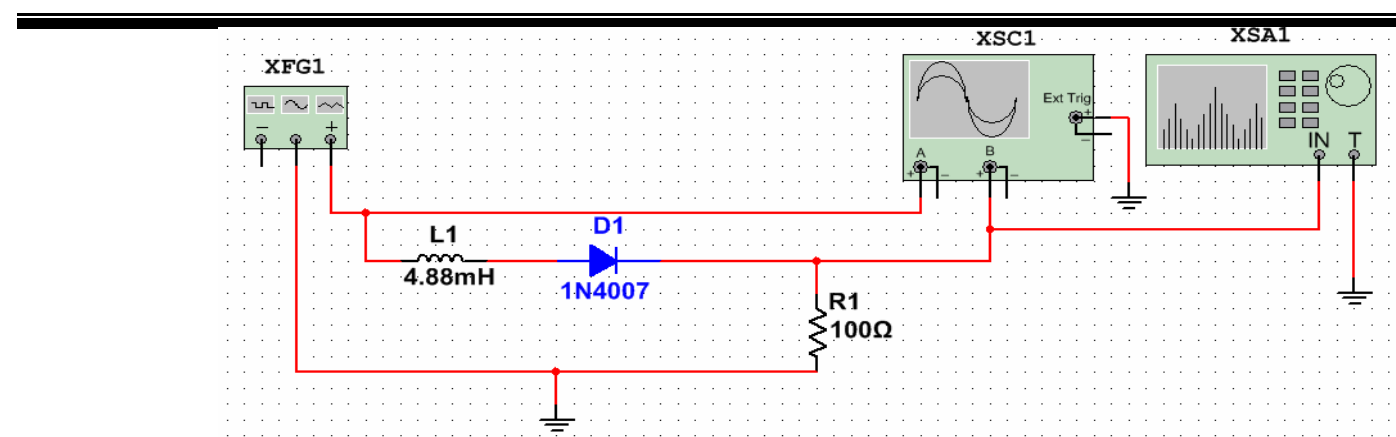

Рисунок 1 - Моделювание RLD цепи в Multisim

Исследование модели RLD цепи в Multisim проводилось в два этапа.

На первом этапе исследовалось влияние амплитуды при ее увеличении и уменьшении $U$ входного сигнала на $\max \left(U_{\mathrm{d}}\right)$. Переход к хаотическому режиму в RLD цепи при увеличении амплитуды входного гармонического сигнала $U$ с

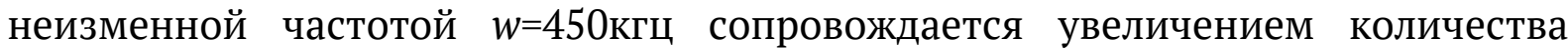
непересекающихся петель фазового портрета и наполнением спектрального диапазона 0-2 $w$ новыми спектральными компонентами. В результате фазовый портрет приобретает вид странного аттрактора, а спектр - становится непрерывным, что служит признаком хаоса (рис.2).

Последовательное увеличение, а затем уменьшение амплитуды $U$ гармонических колебаний показало наличие петли гистерезиса на интервале 350-400 mv. Этот интервал характеризуется возникновением хаотических колебаний.

На втором этапе исследовалось влияние частоты $w$ входного гармонического напряжения при ее увеличении и уменьшении на $\max \left(U_{\mathrm{d}}\right)$ на полупроводниковом диоде. Последовательное увеличение, а затем уменьшение частоты $w$ гармонических колебаний при неизменной амплитуде $U$ показало наличие петли гистерезиса на интервале 40-45 кгц. Этот интервал характеризуется возникновением хаотических колебаний.

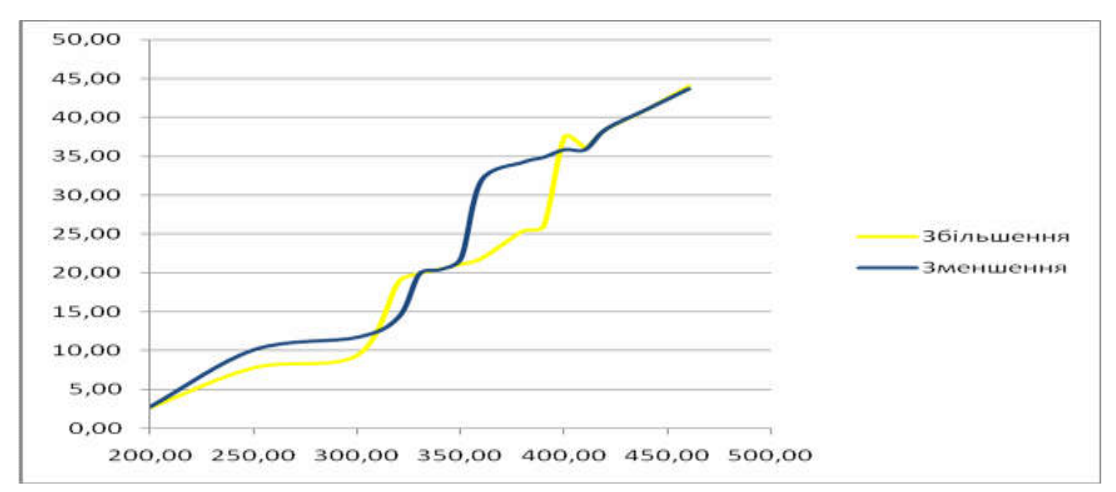

Рисунок 2 - Зависимость $\max (\mathrm{Ud})$ от амплитуды $\mathrm{U}$ гармонических колебаний при неизменной частоте $\mathrm{w}=450$ кгц 


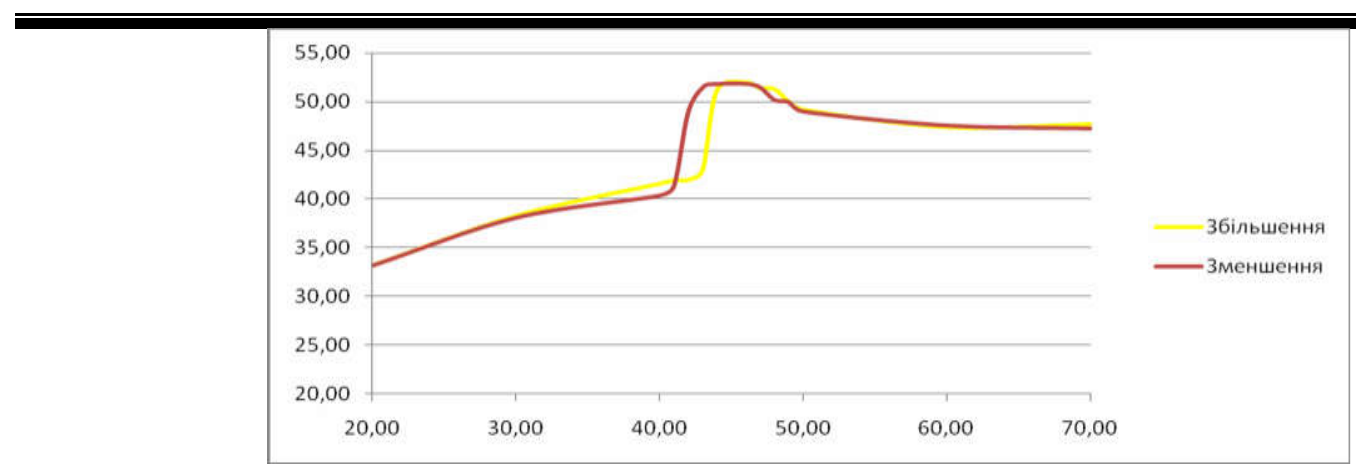

Рисунок 3 - Зависимость $\max (\mathrm{Ud})$ от частоты w гармонических колебаний при неизменной амплитуде U

Таким образом, возникновение хаотических колебаний может быть обусловлено как изменениями амплитуды, так и частоты входного гармонического сигнала. Поэтому важной практической задачей является прогнозирование точек бифуркации петли гистерезиса (рис.3).

\section{Выводы}

Проведенные исследования дали возможность:

во-первых, оценить зависимость $\max \left(U_{\mathrm{d}}\right)$ от частоты $w$ и амплитуды $U$ гармонических колебаний;

во-вторых, получить на основе рассмотренной компьютерной модели возможность прогнозирования точек бифуркации петли гистерезиса для реальных элементов электронных схем.

\section{Литература}

1. Hanias M.P., Giannarias G., Spyridokis A., Rigas A. Time serias analysis in chaotic diode resonator circuit. Chaos, Solitons and Fractals, 27, 2006, 569-573p.

2. Стокер Дж. Нелинейные колебания в механических и электрических системах. Иностранная литература, М. 1953. С.253.

\section{CHAOTIC OSCILLATIONS IN RLD CHAINS}

Derevyanko Aleksandr

Abstract. A diode resonator chaotic circuit is presented. Multisim is used to simulate the circuit and show the presence of chaos.

Keywords: CHAOTIC DYNAMICS, RLD CHAINS, BIFURCATION POINTS.

\section{References}

1. Hanias M.P., Giannarias G., Spyridokis A., Rigas A. Time serias analysis in chaotic diode resonator circuit. Chaos, Solitons and Fractals, 27, 2006, 569-573p.

2. Stoker Dzh. Nelyneinыe kolebanyia v mekhanycheskykh y эlektrycheskykh systemakh. Ynostrannaia lyteratura, M. 1953. S.253. 